\title{
The problem of childhood obesity and the responsibility of primary care providers: a review
}

\author{
Melissa A Wright ${ }^{1 *}$ and Jessica L Jones ${ }^{2}$ \\ ${ }^{1}$ University of Utah School of Medicine, USA \\ ${ }^{2}$ Department of Family and Preventative Medicine, Division of Public Health, USA
}

\section{Introduction}

Obesity-related behaviors are a leading cause of premature, preventable death in the United States, second only to smoking [1]. Obesity is associated with many chronic diseases, including, but not limited to, hypertension, type 2 diabetes mellitus, gallbladder disease, coronary artery disease, and hypercholesterolemia [2]. While these conditions typically affect adults, these comorbidities have become a growing concern in the pediatric population [3].

Rates of obesity are at epidemic levels, and American children have not been exempt. Obese adults strongly influence the risk of childhood obesity, and obese children are at high risk for becoming obese adults [4]. Overweight in children is defined as $\geq 85^{\text {th }}$ percentile for age and sex, while obesity is $\geq 95^{\text {th }}$ percentile and severe obesity is $\geq 99 \%$ ile $[5,6]$. Primary care providers (PCPs) have a great responsibility to help children who suffer from this chronic disease; it is therefore essential that they gain knowledge regarding the topic to effectively manage and treat their patients.

\section{Scope of the problem}

The prevalence of both adult and childhood obesity throughout the world has risen dramatically during the twentieth and twentyfirst centuries. Currently there are an estimated 43 million children under the age of 5 who are overweight or obese, a figure expected to rise to 60 million by 2020 [7]. In 2014, more than 12\% of American kindergarteners were obese, while nearly $15 \%$ were overweight. During that same year, $17 \%$ of eighth graders were overweight, with a startling one-fifth of students categorized as obese [8].

Overall prevalence of high body mass index ( $\geq 85$ percentile of CDC growth charts) for all children ages 2-19 has reached a staggering $32 \%$ [9]. These figure are in stark contrast to past estimates, as prevalence among 6-11 year olds has seen a 5-fold increase since the 1960s $[9,10]$. Fortunately, prevalence of childhood obesity in the U.S. has stabilized at $17 \%$, but remains unacceptably high [9].

Many factors contribute to the obesity epidemic, reflecting the complex nature of the disease itself. Rare single gene defects in the leptin signaling pathway can cause obesity [11]. Genetic syndromes, such as Prader-Willi, WAGR, and Beckwith-Wiedemann, and endocrine disorders, including hypothyroidism, hypothalamic disorders, and cortisol excess, may be causative factors [12]. Obesity can also be iatrogenic from certain medications. However, less than $5 \%$ of cases of childhood obesity can be attributed to a single, identifiable cause [13]. Rather, obesity remains a complicated interplay of genetics, epigenetics, society, behaviors, and environmental factors. Twin studies suggest a high heritability of adiposity [14]. Epidemiologic studies of obesity reveal a higher risk in Hispanic, non-Hispanic black, and American Indian children [15]. Lower prevalence rates of obesity in the wealthiest American families suggest socioeconomic factors [8]. Psychosocial elements are also involved, as obese children are more likely to have undergone a serious family crisis compared to overweight peers [16].

While the above factors attest to the complicated problem of obesity, they do not explain the rapid rise in obesity over the last 50 years. Americans now eat 800 more calories per day than they did 50 years ago [17]. The development of sweet corn-based syrups, increased vegetable oil consumption, food marketing strategies, relatively inexpensive cost of food in America, suppression of health information, and popularity of sugar-sweetened beverages have been speculated as likely factors in this increased intake $[17,18]$. Moreover, behaviors related to reduced energy expenditure, such as duration of television watching, are associated with increased BMIs [19]. The content of TV advertising itself has also been shown to influence the short-term food preferences and requests of children [20]. Others have noted the use of motorized transport and decline in opportunities for physical recreation as challenging trends [21]. Behaviors related to overscheduled lifestyles are also associated with obesity. Frequent family dinners and increased duration of sleep have both been identified as protective against high BMIs [22,23]. Processed and take-out foods often contain ingredients that are calorie dense, and the consumption of fried foods outside the home is associated with weight gain in adolescents [24]. Additionally, restaurant meals and pre-packaged foods often contain multiple serving sizes, and portion size has been shown to influence the amount of food consumed by research participants [25]. Overall, societal trends towards higher caloric intake and lower energy expenditure seem to be a principal source behind the multifactorial obesity problem.

\section{Effects of childhood obesity}

Obesity has implications for individuals, families, societies, and healthcare systems. Childhood obesity is responsible for $\$ 14$ billion in direct costs for outpatient care alone [26]. The complications of childhood obesity mirror those of adult obesity, and obese children have poorer health statuses compared to their peers [3]. Comorbidities of obesity include: sleep apnea, asthma, slipped capital epiphyses, tibial

Correspondence to: Melissa A. Wright, University of Utah School of Medicine, 30 N 1900 E, Salt Lake City, UT 84132, USA, Tel: 630-699-5179; E-mail: Melissa. wright@hsc.utah.edu

Key words: childhood obesity, obesity management, obesity prevention

Received: April 05, 2017; Accepted: May 04, 2017; Published: May 07, 2017 
torsion, increased risk of fractures and sprains, idiopathic intracranial hypertension, NAFLD, and GERD [21,27]. Orthopedic complications are the most common encountered, but metabolic problems are seen in $42 \%$ of obese children and include insulin resistance, type II diabetes, metabolic syndrome, and thyroid dysfunction [27]. One third of obese children have cardiovascular comorbidities such as hyperlipidemia, hypertension, and accelerated atherosclerosis [21,27,28]. PCOS and early menarche may also be seen [21].

Importantly, obesity has psychological impacts on children. Obese children have lower self-worth regarding their physical competence [29], and are subject to more bullying [16]. Anxiety, depression, bulimia, binge eating, ADHD, learning disabilities, and conduct disorders have been more commonly reported in obese children [3]. Furthermore, an estimated $24 \%$ of the variance in academic performance can be accounted for by BMI, physical activity, and diet [30]. Obesity has been shown to contribute to declining motor skills in children, which may have lifelong implications regarding patients' abilities to be physical activity [31]. The comorbidities of obesity follow children into adulthood, and a high BMI is predictive of later morbidity and mortality in adulthood [32].

\section{The role of the Primary Care Provider (PCP)}

Because of the multifactorial component of obesity, the most successful programs for lowering obesity rates in the United States will likely involve schools, communities, health care providers, families, and governmental regulations. PCPs are in the unique position to follow children's health over frequent office visits, to partner with parents and influence family behaviors, and to advocate for policy change. While PCPs alone cannot solve the childhood obesity problem, assessment of obesity and obesity-related problems in children "falls squarely in the purview of health care providers" [6]. At the peak of childhood obesity in 2004, providers identified obesity in only half of obese children, with particularly low identification rates among children under five [33]. More can be done to battle childhood obesity, especially in primary care offices.

\section{Prevention and surveillance}

The widespread consensus among researchers is that prevention is the best way to combat childhood obesity [11]. The means by which this can feasibly occur however, is less clear. Evidence supports the implementation of preventative measure by PCPs from before birth, as maternal obesity, smoking, and weight gain during pregnancy are all risks factors for the development of childhood obesity [34]. PCPs can support obstetricians in relaying messages regarding healthy pregnancy practices to their patients and patients' families. In early infancy, exclusive breastfeeding for the first six months should be encouraged as it reduces the risk for obesity later in life [35].

The early years of a child's life are critical in establishing behaviors that will carry into adulthood. The annual incidence of childhood obesity is highest among younger ages, [8] signifying the importance of early prevention. The AAP recommends that during the period of weaning and preschool age, PCPs should guide parents to establish healthy habits in their children. Sugar-sweetened beverages should not be offered, and $100 \%$ fruit juice should only be given in small portions, if at all. Foods should resemble the same variety as healthy adult diets. Parents should be encouraged to offer new foods multiple times, while avoiding controlling or restrictive food-related practices. PCPs should also counsel parents that children under age 2 should not have solo media use [34].
In older children, the evidence supports a similar focus on modifiable risk factors. Expert committee recommendations support the following behaviors: minimizing sugar-sweetened beverages, eating a diet in line with USDA recommendations, avoiding skipping breakfast, limiting fast food and portion size, having meals as a family, promoting physical activity for 60 minutes a day, and removing screens from the areas that children sleep so that screen time can be monitored [6].

PCPs face many challenges in the fight against childhood obesity. Parents often do not recognize high BMI in their own children, or may not understand associated health risks [36]. They themselves may be obese and feel unmotivated by failed personal attempts. Parental buyin and family support is essential in changing behaviors of children at high risk for developing obesity. Studies support the use of motivation interviewing in assessing readiness for change, identifying goals, and modifying behaviors [37-39] PCPs can help encourage families to stay motivated, offer possible solutions to challenges, and praise successes. Furthermore, discussions should focus on nutrition and physical activity rather than weight [40].

PCPs are responsible for monitoring their patients for risk factors and rising BMIs. At a minimum, BMI should be documented annually for every child ages 2-18 and functions as an initial screen [6]. For children under the age of 2, weight-for-length should be used with WHO growth charts [34]. Practitioners should interpret and act on BMI findings in the context of risks related to medical health, behaviors, and motivation of families [6]. Simple screening questions to parents about their children's diet and physical activity or about their own level of knowledge regarding obesity risk factors can be useful to guide further discussions [34,41]. In a 2007 report, Barlow et al outlines a "fifteenminute obesity prevention protocol". This five-step process includes assessment of BMI and obesity-associated behaviors, setting an agenda to identify target behaviors, assessing motivation and confidence using motivational interviewing techniques, summarizing the plan for change, and scheduling follow up [6]. While this protocol is likely not feasible for every child given time restrictions of office visits, it provides a framework for providers to abbreviate or expand upon based on the child's risk for developing obesity and current BMI.

Primary care providers can also support efforts to combat obesity outside of the office. Behavioral and lifestyle interventions that are family-centered appear to be effective in reducing weight, and PCPs can advocate for such programs in schools and communities [42]. They can also promote government initiatives to encourage healthy behaviors and help change the public's perception of unhealthy foods for children [17]. Training resources such as the AAP's Change Talk website [34] can ease providers' feelings of inexperience in having these discussions with families [43]. Learning about means of obtaining inexpensive access to healthy foods and physical activities can also arm providers with possible solutions to challenges that families face.

\section{Management and treatment}

When prevention fails, PCPs are responsible for managing the disease. Any child with a $\mathrm{BMI} \geq 85^{\text {th }}$ percentile and comorbidities should be treated [11]. The target outcomes of treatment depend on the age and severity of obesity. Younger children or those with relatively lower BMIs may be treated with the goal of weight maintenance. Older children, or younger children with BMIs $>95^{\text {th }}$ percentile, warrant an aim of gradual weight loss. Aggressive weight loss should be reserved for obese children 12 or older, or for severely obese children between the ages of 6-11 [6]. 
The mainstay of treatment is non-medical and focuses on all the same lifestyle modifications that are essential for prevention. Providers should thoroughly assess children for risk factors and associated comorbidities in order to target unhealthy behaviors. Research suggests that neither diet nor exercise alone are as effective as when used in combination [44]. Children cannot be expected to make modifications alone, and have a better chance of being successful when there are household-wide changes. As with prevention, motivational interviewing is central in implementing treatment for children and parents. Office and provider based interventions focusing on motivational interviewing and education of families regarding nutrition and physical activity have been shown to be feasible [45] and effective in changing behaviors $[38,46]$. Reviews of PCP mediated interventions demonstrate that PCP driven obesity treatment can be both successful and sustainable [47].

Medical treatment may be necessary for severely obese children who fail non-pharmacologic management. Research on the use of medication for the treatment of obesity in children is limited. Currently, there are two weight loss medications FDA approved for children. Sibutramine, a serotonin reuptake inhibitor, is approved for children 16 years or older. Orlistat, an intestinal lipase inhibitor, is approved for children 12 years or older. While meta-analysis from randomized controlled data does indicate minor effectiveness of both orlistat and sibutramine when combined with lifestyle interventions, side effects may limit treatment tolerance [42]. Surgical options do exist, and bariatric procedures are effective in decreasing BMI [13]. Because of the paucity of data regarding long-term outcomes and consequences though, bariatric surgery should be reserved for severe cases in adolescents with significant comorbidities. Furthermore, patients need to have failed lifestyle and pharmacologic intervention, as well as have achieved $95 \%$ of adult stature [48]. Overall, more evidence is needed regarding these aggressive treatment options.

Currently, the AMA expert committee recommends that providers adopt a staged protocol in treating childhood obesity, a method that is also supported by both the AAFP and AAP [6,49]. The four identified stages are prevention plus, structured weight management, comprehensive multidisciplinary intervention, and tertiary care intervention. The first three stages center on modifying unhealthy behaviors, with each level adopting more detailed goals regarding diet and exercise, stricter restrictions on intake and sedentary time, and greater reliance on dieticians, exercise specialists, and/or established weight loss programs. The final stage, tertiary care intervention, incorporates medications and possibly surgery into treatment recommendations. Factors such as age, severity of obesity, existence of comorbidities, family readiness, and psychosocial dynamics can help guide the time spent in each stage [6]. The USPSTF recommendations also support the identification of obesity through BMI screening, as well as the use of comprehensive behavioral interventions for treatment [50].

\section{Challenges and the future}

The speed by which the childhood obesity problem has grown appears to have slowed, but much work is needed to reverse the problem. As of now, the data remains mixed, although supportive of interventions that focus on behavioral lifestyle interventions, involve parents, and are tailored to a patient's age and situation. ${ }^{42}$ The heterogeneity of research itself may point towards the need for changes at all levels, including those of individuals, families, schools, communities, social trends, medical personnel, and governments.
As defenders of children's health, primary care providers have a responsibility to prevent, recognize, and manage childhood obesity in their patients.

\section{References}

1. Mokdad AH, Marks JS, Stroup DF, Gerberding JL (2004) Actual causes of death in the United States, 2000. JAMA 291: 1238-1245. [Crossref]

2. Must A, Spadano J, Coakley EH, Field AE, Colditz G, et al. (1999) The disease burden associated with overweight and obesity. JAMA 282: 1523-1529. [Crossref]

3. Halfon N, Larson K, Slusser W (2013) Associations between obesity and Comorbid mental health, developmental, and physical health conditions in a nationally representative sample of US children aged 10 to 17. Academic Pediatrics 13: 6-13.

4. Koplan JP, Dietz WH (1999) Caloric imbalance and public health policy. JAMA 282 1579-1581.

5. Shields M, Tremblay MS (2010) Canadian childhood obesity estimates based on WHO, IOTF and CDC cut-points. Int J Pediatr Obes 5: 265-273.

6. Barlow SE, Expert Committee (2007) Expert committee recommendations regarding the prevention, assessment, and treatment of child and adolescent overweight and obesity: summary report. Pediatrics 120 Suppl 4: S164-192. [Crossref]

7. de Onis M, Blössner M, Borghi E (2010) Global prevalence and trends of overweigh and obesity among preschool children. Am J Clin Nutr 92: 1257-1264. [Crossref]

8. Cunningham SA, Kramer MR, Narayan KM (2014) Incidence of childhood obesity in the United States. N Engl J Med 370: 403-411. [Crossref]

9. Ogden CL, Carroll MD, Kit BK, Flegal KM (2014) Prevalence of childhood and adult obesity in the United States, 2011-2012. JAMA 311: 806-814. [Crossref]

10. CDC, Ogden C, Carroll M (2010) Prevalence of obesity among children and adolescents: United States, trends 1963-1965 through 2007-2008. Center for Disease Control and Prevention.

11. Han JC, Lawlor DA, Kimm SY (2010) Childhood obesity. Lancet 375: 1737-1748. [Crossref]

12. Weiss R, Lustig RH (2014) Obesity, metabolic syndrome, and disorders of energy balance. $\left(4^{\text {th }}\right.$ edn) In: Sperling MA. Pediatric endocrinology. United States: Saunders; pp: 956-1014.

13. Atay Z, Bereket A (2016) Current status on obesity in childhood and adolescence Prevalence, etiology, co-morbidities and management. Obesity Medicine 3: 1-9.

14. Maes HH, Neale MC, Eaves LJ (1997) Genetic and environmental factors in relative body weight and human adiposity. Behavior Geneics 27: 325-351.

15. Gurnani M, Birken C, Hamilton J (2015) Childhood Obesity: Causes, Consequences, and Management. Pediatric Clinics of North America 62: 821-840.

16. Häkkänen P, Ketola E, Laatikainen T (2016) Development of overweight and obesity among primary school children-a longitudinal cohort study. Family Practice 33: 368-373.

17. Perry CL, Creamer MR (2014) The childhood obesity epidemic: lessons learned from tobacco. J Pediatr 164: 178-185. [Crossref]

18. Malik VS, Pan A, Willett WC, Hu FB (2013) Sugar-sweetened beverages and weigh gain in children and adults: a systematic review and meta-analysis. Am J Clin Nutr 98: 1084-1102.

19. Kaur H, Choi WS, Mayo MS, Harris KJ (2003) Duration of television watching is associated with increased body mass index. J Pediatrics 143: 506-511.

20. McGinnis MJ, Gootman JA, Kraak VI (2006) Food marketing to children and youth: Threat or opportunity? Washington, DC: National Academies Press.

21. Lobstein T, Baur L, Uauy R; IASO International Obesity TaskForce (2004) Obesity in children and young people: a crisis in public health. Obes Rev 5 Suppl 1: 4-104. [Crossref]

22. Wansink B, van Kleef E (2013) Dinner rituals that correlate with child and adult BMI Obesity 22: 91-95.

23. Patel SR, Hu FB (2008) Short sleep duration and weight gain: a systematic review. Obesity (Silver Spring) 16: 643-653. [Crossref]

24. Taveras EM, Berkey CS, Rifas-Shiman SL, Ludwig DS, Rockett HR, et al. Association of Consumption of Fried Food Away From Home With Body Mass Index and Diet Quality in Older Children and Adolescents. Pediatrics 116: e518-e524. 
25. Division of Nutrition and Physical Activity (2006) Research to Practice Series No. 2: Portion Size. Atlanta: Centers for Disease Control and Prevention.

26. Trasande L, Chatterjee S (2009) The impact of obesity on health service utilization and costs in childhood. Obesity 17: 1749-1754.

27. Maggio AB, Martin XE, Saunders Gasser C, Gal-Duding C, Beghetti M, et al. (2014) Medical and non-medical complications among children and adolescents with excessive body weight. BMC Pediatrics 14: 232

28. Freedman DS, Patel DA, Srinivasan SR, Chen W, Tang R, et al. (2008) The contribution of childhood obesity to adult carotid intima-media thickness: the Bogalusa Heart Study. Int J Obes (Lond) 32: 749-756. [Crossref]

29. Braet C, Mervielde I, Vandereycken W (1997) Psychological aspects of childhood obesity: a controlled study in a clinical and nonclinical sample. J Pediatr Psychol 22: 59-71. [Crossref]

30. Sigfusdottir ID, Kristjansson AL, Allegrante JP (2006) Health behaviour and academic achievement in Icelandic school children. Health Education Research 22: 70-80.

31. Cheng J, East P, Blanco E, Sim EK, Castillo M, et al. (2016) Obesity leads to declines in motor skills across childhood. Child Care Health Dev 42: 343-350.

32. Must A, Strauss RS (1999) Risks and consequences of childhood and adolescent obesity. Int J Obes (Lond) 23: S2-S11.

33. O’Brien SH, Holubkov R, Reis EC (2004) Identification, Evaluation, and Management of Obesity in an Academic Primary Care Center. Pediatrics 114: e154-e159.

34. Daniels S, Hassink SG, Committee on Nutrition (2015) The Role of the Pediatrician in Primary Prevention of Obesity. Pediatrics 136: e275-e292.

35. Weyermann M, Rothenbacher D, Brenner H (2006) Duration of breastfeeding and risk of overweight in childhood: a prospective birth cohort study from Germany. Int J Obes (Lond) 30: 1281-1287.

36. Eckstein KC, Mikhail LM, Ariza AJ, Thomson JS, Millard SC, et al. (2006) Parents' perceptions of their child's weight and health. Pediatrics 117: 681-690. [Crossref]

37. Schwartz RP, Hamre R, Dietz WH, Wasserman RC, Slora EJ, et al. (2007) OfficeBased Motivational Interviewing to Prevent Childhood Obesity. Arch Pediatr Adolesc Med 161: 495-501.

38. Taveras EM, Gortmaker SL, Hohman KH, Horan CM, Kleinman KP, et al. (2011) Randomized controlled trial to improve primary care to prevent and manage childhood obesity: the High Five for Kids study. Arch Pediatr Adolesc Med 165: 714-722.
39. Resnicow K, McMaster F, Bocian A, Harris D, Zhou Y, et al. (2015) Motivational interviewing and dietary counseling for obesity in primary care: an RCT. Pediatrics 135: 649-657. [Crossref]

40. Perrin EM, Finkle JP, Benjamin JT (2007) Obesity prevention and the primary care pediatrician's office. Curr Opin Pediatr 19: 354-361.

41. Fitch A, Fox C, Bauerly K, Gross A, Heim C, et al. (2013) Institute for Clinical Systems Improvement. Prevention and Management of Obesity for Children and Adolescents.

42. Oude Luttikhuis H, Baur L, Jansen H, Shrewsbury VA, O’Malley C, et al. (2009) Interventions for treating obesity in children. Cochrane Database Syst Rev 1: CD001872?

43. Jelalian E, Boergers J, Alday CS, Frank R (2003) Survey of physician attitudes and practices related to pediatric obesity. Clinical Pediatrics 42: 235-245.

44. Ben Ounis O, Elloumi M, Lac G, Makni E, Van Praagh E, et al. (2009) Two-month effects of individualized exercise training with or without caloric restriction on plasma adipocytokine levels in obese female adolescents. Ann Endocrinol (Paris) 70: 235-241. [Crossref]

45. Schwartz RP, Hamre R, Dietz WH, Wasserman RC, Slora EJ, et al. (2007) Officebased Motivational interviewing to prevent childhood obesity. Arch Pediatr Adolesc Med 161: 495-501.

46. Siwik V, Kutob R, Ritenbaugh C, Cruz L, Senf J, et al (2013) Intervention in overweigh children improves body mass index (BMI) and physical activity. J Am Board Fam Med 26: 126-137.

47. Bhuran SS, Chandak A, Smith P, Carlton EL, Duncan K, et al. (2015) Integration of public health and primary care: A systematic review of the current literature in primary care physician mediated childhood obesity interventions. Obesity Research Clinical Practice 9: 539-552.

48. Hsia DS, Fallon SC, Brandt ML (2012) Adolescent Bariatric surgery. Arch Pediatr Adolesc Med 166: 757-766.

49. Rao G (2008) Childhood obesity: highlights of AMA Expert Committee recommendations. Am Fam Physician 78: 56-63.

50. Whitlock EP, O'Connor EA, Williams SB, Beil TL, Lutz KW (2010) Effectiveness of weight management interventions in children: A targeted systematic review for the USPSTF. Pediatrics 125: e396-e418.

Copyright: (C2017 Wright MA. This is an open-access article distributed under the terms of the Creative Commons Attribution License, which permits unrestricted use, distribution, and reproduction in any medium, provided the original author and source are credited. 